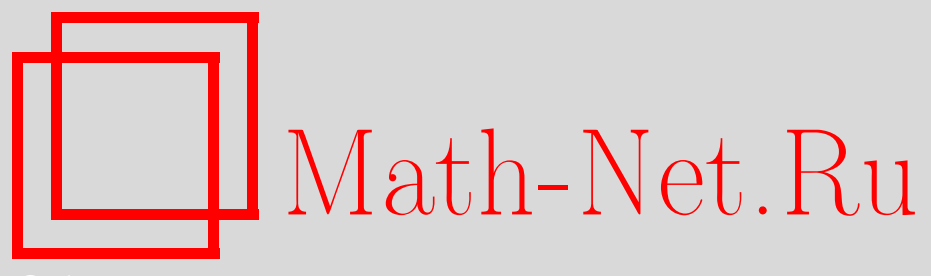

Е. М. Варфоломеев, О нормальности некоторых эллиптических функционально-дифференциальных операторов второго порядка, УМН, 2006, том 61, выпуск 1, 173174

DOI: https://doi.org/10.4213/rm1708

Использование Общероссийского математического портала Math-Net.Ru подразумевает, что вы прочитали и согласны с пользовательским соглашением http://www . mathnet.ru/rus/agreement

Параметры загрузки:

IP : 3.80 .253 .173

26 апреля 2023 г., 06:39:33

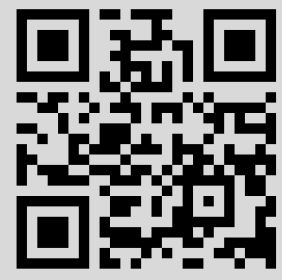




\section{О нормальности некоторых эллиптических функционально-дифференциальных операторов второго порядка}

\section{Е. М. Варфоломеев}

В нелинейных оптических системах с преобразованием поля в двумерной обратной связи возникают различные регулярные периодические явления, которые называют "многолепестковыми волнами" [1], [2]. Эти световые структуры используются в современных компьютерных технологиях для создания оптических аналогов нейронных сетей. Математическая модель указанной системы описывается бифуркацией периодических решений квазилинейного параболического функционально-дифференциального уравнения с преобразованием пространственных переменных $g(x)$. В работах [3], [4] эта задача рассматривалась в случае, когда область $Q-$ круг или кольцо, а преобразование $g$ - вращение на некоторый угол $\theta$. Случай, когда область $Q \subset \mathbb{R}^{2}$ и преобразование $g$ произвольны, рассматривался в работах [5], [6]. В этих статьях предполагалось, что линеаризованный эллиптический функционально-дифференциальный оператор - нормальный. В работе [7] были получены необходимые и достаточные условия нормальности указанного оператора в терминах области $Q \subset \mathbb{R}^{n}$ и преобразования $g$. Более общий случай без предположения нормальности линеаризованного эллиптического оператора рассмотрен в работе [8].

В настоящей работе получены необходимые и достаточные условия нормальности линеаризованного оператора в случае двух преобразований пространственных переменных.

Пусть $Q \subset \mathbb{R}^{n}$ - ограниченная область с границей $\partial Q \subset C^{\infty}, n \geqslant 2$. Пусть $g, f-$ взаимно однозначные преобразования класса $C^{3}$ такие, что

$$
\begin{array}{lll}
g: V \subset \mathbb{R}^{n} \rightarrow g(V) \subset \mathbb{R}^{n}, & \left|J_{g}(x)\right| \neq 0, & x \in V ; \\
f: V \subset \mathbb{R}^{n} \rightarrow f(V) \subset \mathbb{R}^{n}, & \left|J_{f}(x)\right| \neq 0, & x \in V .
\end{array}
$$

Здесь $V$ - ограниченная область, $\bar{Q} \subset V, J_{g}(x)=\left[\partial g_{i} / \partial x_{j}\right]_{i, j=1}^{n}$ - матрица Якоби преобразования $g,\left|J_{g}(x)\right|=\left|\operatorname{det} J_{g}(x)\right|$. Пусть также выполнено $g(Q) \subset Q$ и $f(Q) \subset Q$.

Рассмотрим неограниченный оператор $A_{0}: L_{2}(Q) \rightarrow L_{2}(Q)$ с областью определения

$$
\mathscr{D}\left(A_{0}\right)=\left\{v \in W_{2}^{2}(Q): B v=0\right\},
$$

действующий по формуле $A_{0} v=\Delta v, v \in \mathscr{D}\left(A_{0}\right)$. Здесь $W_{2}^{k}(Q)$ - пространство Соболева комплекснозначных функций, принадлежащих $L_{2}(Q)$ вместе со всеми обобщенными производными до порядка $k$ включительно, $B v=\left.v\right|_{\partial Q}$ или $B v=\left.(\partial v / \partial \nu)\right|_{\partial Q}$, $\nu$ - единичный вектор внутренней нормали к $\partial Q$ в точке $x \in \partial Q$. Как известно, $A_{0}$ - самосопряженный оператор. Положим $A: L_{2}(Q) \rightarrow L_{2}(Q), A=A_{0}+A_{1}+A_{2}$, где $A_{1}, A_{2}$ - линейные ограниченные операторы, определенные на всем пространстве $L_{2}(Q)$ следующим образом:

$$
\begin{array}{ll}
A_{1}: L_{2}(Q) \rightarrow L_{2}(Q), & A_{1} v(x)=a_{1} v(g(x)), \\
A_{2}: L_{2}(Q) \rightarrow L_{2}(Q), & A_{2} v(x)=a_{2} v(f(x)),
\end{array}
$$

где $a_{1} \neq 0, a_{2} \neq 0$ - вещественные числа.

Оператор $A$ называется нормальным, если $\mathscr{D}\left(A A^{*}\right)=\mathscr{D}\left(A^{*} A\right)$ и $A A^{*} v=A^{*} A v$ для любого $v \in \mathscr{D}\left(A^{*} A\right)$. Положим $D(A)=D\left(A_{0}\right)$.

Определим множества $G_{g}^{m}=\left\{x \in Q: g^{m}(x) \neq x\right\}, m=1,2, \ldots$. Здесь $g^{m}(x)$ обозначает преобразование $g$, примененное $m$ раз. Обозначим $\widetilde{G}_{g}^{m}=Q \backslash G_{g}^{m}$. Будем также записывать суперпозицию преобразований в виде $f g(x), g^{-1} f(x)$ и т. п.

TEOPEMA 1.

Работа выполнена при поддержке РФФИ (грант № 04-01-00256) и Министерства образования и науки РФ (грант № УР.04.01.129). 
Основными результатами данной работы являются следующие теоремы.

Пусть $G_{g}^{2} \neq \varnothing, G_{f}^{2} \neq \varnothing$. Кроме того, пусть $g(Q)=f(Q)=Q u\left|a_{1}\right| \neq\left|a_{2}\right|$. Тогда для нормальности оператора $A$ необходимо и достаточно, чтобъ:

$$
\begin{gathered}
g(x)=K x+b, \quad f(x)=C x+d, \quad x \in Q, \\
g f(x)=f g(x), \quad x \in Q,
\end{gathered}
$$

где $K, C$ - ортогональные матрицы порядка $n \times n, K^{2} \neq E, C^{2} \neq E, b, d \in \mathbb{R}^{n}$.

Теорема 2. Пусть $G_{g}^{2}=\varnothing, G_{f}^{2}=\varnothing$. Тогда $g(Q)=f(Q)=Q$, а также:

1) если оператор $A$ - нормальный и выполнено $a_{1}+a_{2} \neq 0$, то

$$
\begin{cases}a_{1}^{2}\left(\left|J_{g}(x)\right|-\left|J_{g}(x)\right|^{-1}\right)+a_{2}^{2}\left(\left|J_{f}(x)\right|-\left|J_{f}(x)\right|^{-1}\right)=0, & x \in G_{g}^{1} \cap G_{f}^{1}, \\ \left|J_{g}(x)\right|=\left|J_{f}(x)\right|=1, & x \in Q \backslash\left(G_{g}^{1} \cap G_{f}^{1}\right) ;\end{cases}
$$

2) если $\left|J_{g}(x)\right|=\left|J_{f}(x)\right|=1, x \in Q$, то $A$ - нормалъный самосопряженный onepamop.

Теорема 3. Пусть $G_{g}^{2} \neq \varnothing, G_{f}^{2}=\varnothing$. Кроме того, пусть $g(Q)=Q$. Тогда $f(Q)=Q$, и для нормальности оператора $A$ необходимо и достаточно, чтобъ

$$
\begin{gathered}
g(x)=K x+b, \quad\left|J_{f}(x)\right|=1, \quad x \in Q, \\
g f(x)=f g(x), \quad x \in Q,
\end{gathered}
$$

где $K$ - ортогоналъная матрица порядка $n \times n, K^{2} \neq E, b \in \mathbb{R}^{n}$.

Автор глубоко благодарен профессору А.Л. Скубачевскому за внимание к этой работе и ценные советы.

\section{Список литературы}

[1] М. А. Воронцов, Ю. Д. Думаревский, Д. В. Пруидзе, В.И. Шмальгаузен, Изв. АН СССР. Сер. физ., 52:2 (1988), 374-376. [2] M. A. Vorontsov, N. G. Iroshnikov, R. L. Abernathy, Chaos Solitons Fractals, 4:8-9 (1994), 1701-1716. [3] А. В. Разгулин, Журн. вычисл. матем. и матем. физики, 33:1 (1993), 69-80. [4] A. V. Razgulin, "Rotational multipetal waves in optical system with 2D feedback", Chaos in Optics, Proc. SPIE, 2039, ed. R. Roy, 1993, 342352. [5] А. Л. Скубачевский, УМH, 51:1 (1996), 169-170. [6] А. L. Skubachevskii, Nonlinear Anal., 32:2 (1998), 261-278. [7] А. Л. Скубачевский, Функu. анализ и его прил., 31:4 (1997), 60-65. [8] А. Л. Скубачевский, Дифферени. уравнения, 34:10 (1998), 1394-1401.

\section{Е. М. Варфоломеев (Е. M. Varfolomeev)}

Российский университет дружбы народов

E-mail: varfolomeyev@mail.ru
Представлено Л.Р. Волевичем Принято редколлегией 26.01.2006 